\title{
Photonic Crystal Waveguides
}

\author{
Dmitry Usanov and Alexander Skripal \\ Additional information is available at the end of the chapter
}

http://dx.doi.org/10.5772/intechopen.76797

\begin{abstract}
The original results of theoretical and experimental studies and the properties of microwave one-dimensional waveguide photonic crystals have been generalized. Methods for describing the electrodynamic characteristics of photonic crystals and their relationship with the parameters of periodic structures filling the waveguides have been presented. The results of an investigation on the characteristics of microwave waveguide photonic crystals made in the form of dielectric matrices with air inclusions have been presented. The model of effective dielectric permittivity has been proposed for describing the characteristics of the investigated photonic crystals containing layers with a large number of air inclusions. New types of microwave low-dimensional waveguide photonic crystals containing periodically alternating elements that are sources of higher type waves have been described. The possibility of effective control of the amplitude-frequency characteristics of microwave photonic crystals by means of electric and magnetic fields has been analyzed. Examples of new applications of waveguide photonic crystals in the microwave range have been given: the measuring parameters of the materials and semiconductor nanostructures that play the role of the microwave photonic crystals' periodicity defect; the resonators of near-field microwave microscopes; small-sized matched loads for centimeter and millimeter wavelength ranges on the basis of microwave photonic crystals.
\end{abstract}

Keywords: microwave photonic crystals, forbidden bands, defect mode, electrically controlled characteristics, measurement of micro- and nanostructures, microwave matched loads, low-dimensional photonic crystal

\section{Introduction}

The idea of the possibility of a continuous spectrum of electron energies decay into a set of alternating allowed and forbidden bands in the direction of electron motion and wave propagation in the presence of spatial periodicity of the deformation field was for the first 
time proposed by Keldysh [1]. Periodic semiconductor structures with predetermined parameters of layers were called semiconductor superlattices. The wide interest to the problem of their creation appeared after the article was published in 1970 by Esaki and Tsu, who proposed to make such structures by changing the doping or composition of the layers [2]. The periods in such structures had values from 5 to $20 \mathrm{~nm}$. The number of layers reached several hundred.

The author [3] gave the definition of photonic crystals as materials whose crystal lattice has a periodicity of the permittivity leading to the appearance of the "forbidden" frequencies range, called the photonic band gap. Yablonovich [4] and John [5] proposed to create structures with a photonic band gap, which can be considered as an optical analog of the band gap in semiconductors. In this case, the forbidden band is the frequency range in which the existence of light in the inner part of the crystal is forbidden. The type of defect or disturbance of periodicity in this instance can be different. Such structures have to be created artificially in contrast to natural crystals. In this case, the size of the basic unit element of a photonic crystal should be comparable with the light wavelength. The manufacturing of such structures involves the use of electron-beam and X-ray lithography [6].

As the advantage of such photonic crystals, the author [3] notes the possibility of an exact description of their properties that coincide with the experiment, in contrast to superlattices.

Structures with spatial periodicity of elements were also used in the microwave range to reduce the phase velocity of the wave in comparison with the speed of light in special waveguides, called delay-line structures [7]. The authors of [7] called them "a kind of artificial crystals, the cells of which have large sizes."

Delay-line structures are used in various types of vacuum microwave electronics devices. The specificity of the delay-line systems is the choice of their basic elements from metals and the need to take into account in the design the possibility of passing the electron beam interacting with the field. Examples of periodic structures used in delay-line systems are "meander," "counterpins," systems with alternating diaphragms, and so on.

In the microwave range, the photonic crystal can be realized both on waveguides with dielectric filling $[8,9]$ and on flat transmission lines with periodically changing stripe structure [10]. There are examples of the creation of photonic crystals in the optical, infrared, ultraviolet, microwave ranges. Creating a photonic crystal for the microwave range is the simplest. It should be noted that in the theoretical description of the properties of such structures, unlike, for example, from superlattices, it is not necessary to take into account the properties of transition layers, quantum size effects, the specificity of technological processes. This opens the possibility to more accurately examine the properties of photonic crystals associated with periodicity and, in particular, to use the results of a theoretical description to measure the parameters of their layers as a result of solving the corresponding inverse problem.

Materials with the properties of photonic crystals are also known in nature. They include, for example, noble opal [11], spicules of natural biomineral crystals, the basal spicules of glass sea sponges [12]. The time-varying forbidden band for the frequency region in the 
vicinity of $6 \mathrm{GHz}$ was observed in a solution with a chemical self-oscillating BriggsRauscher reaction characterized by the presence of periodically located regions with different permittivities [13].

\section{One-dimensional microwave photonic crystals based on rectangular waveguides}

\subsection{Microwave photonic crystals: structures with forbidden bands}

The results of theoretical analysis on the characteristics of one-dimensional microwave photonic crystals made on the basis of a rectangular waveguide and their experimental investigation are given, for example, in [14]. The one dimensionality of the crystal means that the dielectric structure that fills the waveguide has a periodicity of dielectric permittivity in one direction (along the wave propagation direction, along the $\mathrm{Z}$ axis). The authors of [14] studied structures in the form of alternating layers with high dielectric permittivity $h$ and low $l(h l-$ pairs) and layers of the $h_{1} l h_{2}$ type ( $h_{1}$ and $h_{2}$ are dielectrics with different permittivities). In the theoretical description, the scattering matrix method was used. The arrangement of the dielectric layers in the waveguide can be characterized by the following relationships:

$$
\begin{gathered}
\varepsilon(x, y, z+d)=\varepsilon(x, y, z), \quad 0 \leq x \leq a, \quad 0 \leq y \leq b, z<\infty, \\
\varepsilon(x, y, z+d)=\left\{\begin{array}{l}
\varepsilon_{1}=\varepsilon_{0} \varepsilon_{r 1} \text { for }-w \leq z \leq 0 \\
\varepsilon_{2}=\varepsilon_{0} \varepsilon_{r 2} \text { for } 0 \leq z \leq v
\end{array} .\right.
\end{gathered}
$$

Here $\varepsilon_{r 1}$ and $\varepsilon_{r 2}$ are the relative permittivities of the two materials and $\varepsilon_{0}$ is the dielectric constant of the vacuum, $d=v+w$.

When continuity conditions are used on surfaces $z=0, z=v$ and $z=-w$ for tangential field components, the equation for finding the wave number $\gamma$ is obtained:

$$
\gamma=\frac{1}{v+w} \arccos \left(L_{T E_{10}^{z}}\right)
$$

where

$L_{T E_{10}^{z}}=\cos \left(\beta_{1} w\right) \cos \left(\beta_{2} v\right)-\frac{\beta_{1}^{2}+\beta_{2}^{2}}{2 \beta_{1} \beta_{2}} \sin \left(\beta_{1} w\right) \sin \left(\beta_{2} v\right), \beta_{1,2}^{2}=\omega^{2} \mu_{0} \varepsilon_{1,2}+k^{2}, k^{2}=(\pi / a)^{2}$.

The authors of [14] proposed to obtain reflection and transmission coefficients using the generalized scattering matrix, which is a result of the application of continuity conditions on media boundaries. The results of measurements and calculation of the transmission coefficient $\left(S_{21}\right)$, characterized by the presence of a forbidden band for microwave photonic crystals consisting of 10 and 20 separate two-layer elements, are presented in [14]. The authors of [14] refer some of the differences between experiment and theory [14] to the imperfection of the waveguide walls. 
To calculate the reflection $S_{11}$ and the transmission $S_{21}$, coefficients of the electromagnetic wave at its normal incidence on an $N$ layer structure, a matrix of wave transfer between regions with different values of wave propagation constants, can be used, similar to [15-17]:

$$
\mathbf{T}\left(z_{j, j+1}\right)=\left(\begin{array}{ll}
\frac{\gamma_{j+1}+\gamma_{j}}{2 \gamma_{j+1}} e^{\left(\gamma_{j+1}-\gamma_{j}\right) z_{j, j+1}} & \frac{\gamma_{j+1}-\gamma_{j}}{2 \gamma_{j+1}} e^{\left(\gamma_{j+1}+\gamma_{j}\right) z_{j, j+1}} \\
\frac{\gamma_{j+1}-\gamma_{j}}{2 \gamma_{j+1}} e^{-\left(\gamma_{j+1}+\gamma_{j}\right) z_{j, j+1}} & \frac{\gamma_{j+1}+\gamma_{j}}{2 \gamma_{j+1}} e^{-\left(\gamma_{j+1}-\gamma_{j}\right) z_{j, j+1}}
\end{array}\right)
$$

which connects the coefficients $A_{j}, B_{j}$ and $A_{j+1}, B_{j+1}$, which determine the amplitudes of the incident and reflected waves on both sides of the boundary $z_{j, j+1}$, by the relation:

$$
\left(\begin{array}{c}
A_{j+1} \\
B_{j+1}
\end{array}\right)=\mathbf{T}\left(z_{j, j+1}\right) \cdot\left(\begin{array}{c}
A_{j} \\
B_{j}
\end{array}\right)
$$

The coefficients $A_{N+1}$ and $B_{0}$, which determine the amplitudes of the wave transmitted through the multilayer structure (Figure 1) and the wave reflected from it, are connected to the coefficient $A_{0}$, determining the amplitude of the incident wave, by the following relation:

$$
\left(\begin{array}{c}
A_{N+1} \\
0
\end{array}\right)=\mathbf{T}_{N} \cdot\left(\begin{array}{c}
A_{0} \\
B_{0}
\end{array}\right)
$$

where

$$
\mathbf{T}_{N}=\left(\begin{array}{ll}
\mathbf{T}_{N}[1,1] & \mathbf{T}_{N}[1,2] \\
\mathbf{T}_{N}[2,1] & \mathbf{T}_{N}[2,2]
\end{array}\right)=\prod_{j=N}^{0} \mathbf{T}_{j,(j+1)}=\mathbf{T}\left(z_{N, N+1}\right) \cdot \mathbf{T}\left(z_{N-1, N}\right) \ldots \mathbf{T}\left(z_{1,2}\right) \cdot \mathbf{T}\left(z_{0,1}\right)
$$

a transmission matrix of an $N$ layer structure.

The reflection $S_{11}=\frac{B_{0}}{A_{0}}$ and transmission $S_{21}=\frac{A_{N+1}}{A_{0}}$ coefficients of an electromagnetic wave interacting with a layered structure are determined by the following relationships:

$$
S_{11}=-\frac{\mathbf{T}_{N}[2,1]}{\mathbf{T}_{N}[2,2]}, S_{21}=\frac{\mathbf{T}_{N}[1,1] \cdot \mathbf{T}_{\mathbf{N}}[2,2]-\mathbf{T}_{N}[1,2] \cdot \mathbf{T}_{N}[2,1]}{\mathbf{T}_{N}[2,2]}
$$

When calculating $S_{11}$ and $S_{21}$, we used the matrixes of wave transfer between regions with different values of wave propagation constants $\gamma_{j}$ and $\gamma_{j+1}$.

The following expressions are used to calculate the reflection $\left|S_{11}\right|^{2} \mid$ and transmission $\left|S_{12}\right|^{2}$ coefficients of the electromagnetic wave expressed in terms of power (in $\mathrm{dB}$ unit):

$$
\left|S_{11}\right|^{2}=10 \log \left|S_{11}\right|^{2},\left|S_{21}\right|^{2}=10 \log \left|S_{21}\right|^{2}
$$




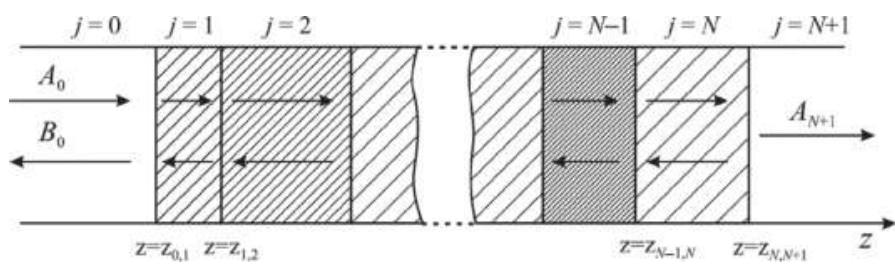

Figure 1. Structure consisting of $N$ layers.

The results of the theoretical and experimental investigation of the resonant features that appear in the allowed and forbidden bands of microwave photonic crystal in the case of creating periodicity disturbance are given in [18]. As a waveguide photonic crystal, a waveguide section with a structure that is periodically alternating layers of two types of dielectrics with different values of thickness and permittivity was used. The dimensions and materials of the layers were chosen in such a way that in the frequency range 8-12 GHz, two allowed and one forbidden band were observed for the propagation of electromagnetic waves. The parameters of the first and the last layers of the photonic crystal were the same. The results of calculating the power transmission coefficient $\left|S_{12}\right|^{2}$ of an electromagnetic wave using the abovementioned relationships for the 11-layer photonic crystal without disturbances in the case of $H_{10}$ wave propagation are shown in Figure 2 (curve 1).

From the results of the calculation presented in Figure 3, it follows that an increase of layers in number causes the decrease of the width of the first band gap completely located within the $3 \mathrm{~cm}$ wavelength range and the increase in the width of both the left and right allowed bands in this wavelength range. With a number of layers larger than 27, these changes are less than $10 \mathrm{MHz}$. This behavior of the characteristics of an electromagnetic propagation through a photonic crystal is due to the following circumstance. The allowed band has a "dissected" frequency response and consists of a set of resonances, the number of which is determined by the number of identical elements that form the photonic crystal. Therefore, the increase of the number of photon crystal layers causes the increase of the number of resonances determining the width of the allowed band and, consequently, its width increases. At the same time, the

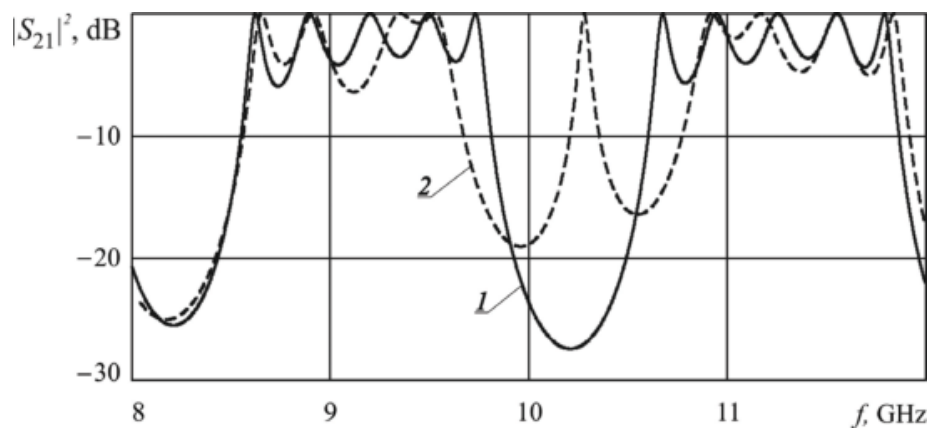

Figure 2. The results of calculating the square of the modulus of the transmission coefficient of an electromagnetic wave through the 11-layer structure without disturbance (curve 1) and with the disturbed central layer (curve 2). 


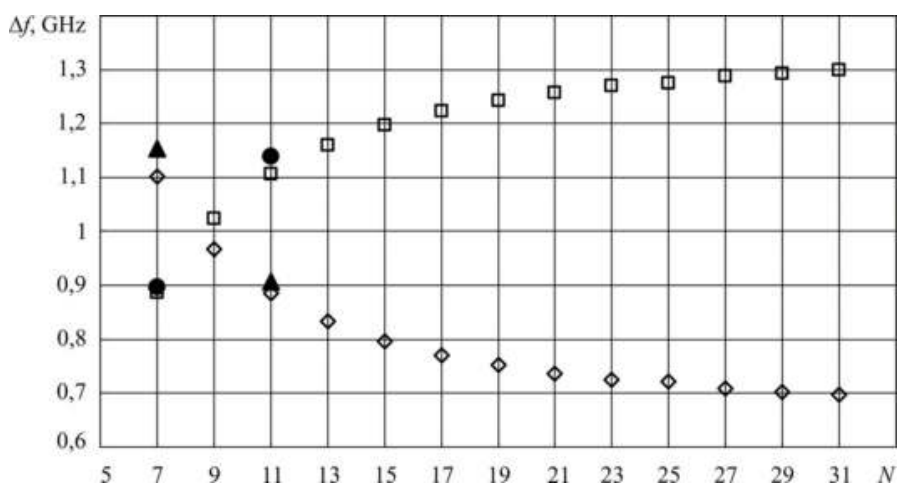

Figure 3. Dependences of the width of the forbidden and left allowed bands on the number of layers of a photonic crystal without disturbance: $\square, \bullet-$ theoretical and experimental values of the width of the left-allowed band, $\diamond, \boldsymbol{\Delta}$-theoretical and experimental values of the band gap width.

width of the forbidden band decreases. The Q-value of the resonances that form the allowed band, the so-called "allowed levels," as follows from the results of calculations and experiments, is maximum near the lower and upper boundaries of the allowed band.

Figure 2 (curve 2) shows the calculations results of the frequency dependences of the square of the modulus of the electromagnetic wave transmission coefficient $S_{21}$ in the presence of a disturbance in the photonic crystal in the form of, for example, a central layer of smaller thickness $d_{6}$. In this case, a resonance feature with a transmission coefficient close to unit appears in the band gap. This feature is an impurity mode resonance whose location can be controlled by varying the dielectric layer thickness. The disturbance in a photonic crystal, as shown in [5], also leads to a change in the number of resonances formed in the allowed band and their positions on the frequency axis. When disturbance in the form of a central layer of a smaller thickness is created in a photonic crystal, the number of resonances in the allowed band decreases by one in comparison with the photonic crystal without disturbance, and the width of the forbidden band containing the impurity mode resonance increases substantially (Figure 2, curve 2). This effect is especially evident for a small number of photonic crystal periods. It should be noted that the width of the forbidden band containing an impurity mode resonance with a number of layers tending to infinity turns out to be approximately equal to the width of the forbidden band of a photonic crystal without disturbances.

The one-dimensional waveguide photonic crystals consisting of 7 and 11 layers completely filling a cross-section of rectangular waveguide of a 3-cm wavelength were used in the experiments. The odd layers were made of $\mathrm{Al}_{2} \mathrm{O}_{3}$ ceramics $\left(\varepsilon_{r}=9.6\right)$, and the even ones were made of Teflon $\left(\varepsilon_{r}=2.1\right)$. The length of odd segments is $1 \mathrm{~mm}$ and the length of even segments is $44 \mathrm{~mm}$. The disturbance was created by changing the length of the central layer, which led to the appearance of an impurity mode resonance in the forbidden band of a photonic crystal. The length of the central disturbed (Teflon) layer was chosen equal to $14 \mathrm{~mm}$. The frequency dependences of the reflection and transmission coefficients of microwave radiation interacting with a photonic 
crystal were measured with the Agilent PNA-L Network Analyzer N5230A. A comparison of the results of calculation and experiment shows their good quantitative coincidence.

In [19], the results of an investigation on the characteristics of a waveguide microwave photonic crystal made in the form of dielectric matrices with air inclusions are presented. Ceramics $\left(\mathrm{Al}_{2} \mathrm{O}_{3}\right)$ with a large number of air inclusions and polystyrene were used as materials of dielectric layers. A waveguide photonic crystal consisting of 11 layers was studied in the frequency range 8-12 GHz (Figure 4a). Odd layers were made of $\mathrm{Al}_{2} \mathrm{O}_{3}$ ceramics $\left(\varepsilon_{r}=9.6\right)$, even layers were made of polystyrene $\left(\varepsilon_{r}=1.05\right)$. Thickness of odd lengths was $d_{\mathrm{Al}_{2} \mathrm{O}_{3}}=1.0 \mathrm{~mm}$, even $d_{\text {foam }}=13.0 \mathrm{~mm}$. The layers completely filled the cross-section of the waveguide. In ceramic layers, a large number of air inclusions were created in the form of square-through holes, which form a periodic structure in the plane of the layer (Figure $4 b$ ).

On the basis of numerical modeling using the finite element method in the CAD ANSYS HFSS, the influence of the volume fraction of air inclusions on the amplitude-frequency characteristics of the transmission coefficient of a photonic crystal was studied. The volume fraction of air inclusions was controlled by changing the size of the holes $a_{\text {hole }}$ in the ceramics plates with a fixed amount of 36 in each of the plates. The simulation was carried out for three sizes of holes, equal to $0.75,1.2$ and $1.65 \mathrm{~mm}$, which corresponds to a volume fraction of air inclusions equal to $8.5,23$ and $43 \%$, respectively. The disturbance of the periodicity of the photonic structure was created by changing the thickness of the central (sixth) polystyrene layer. The thickness of the sixth modified (disturbed) polystyrene layer was 2.25, 2.75, 3.49 and $6.0 \mathrm{~mm}$.

As follows from the results of numerical calculation of the amplitude-frequency characteristics of the transmission coefficient of a photonic crystal by the finite-element method in the absence of the periodicity disturbance in the photonic structure, the increase in the volume fraction of air inclusions in the ceramic layers leads to the shift of the forbidden band of the photonic crystal toward shorter wavelengths and to the decrease of its depth. The results of the calculation of the photonic crystal amplitude-frequency characteristics by the finite element method in the presence of the disturbance of the photonic structure periodicity in the form of a central polystyrene layer of different thickness are shown in Figure 5 (solid curves).

The presence of the disturbance of the photonic structure periodicity in the form of the change of the thickness of the central (sixth) polystyrene layer led to the appearance of a transmission
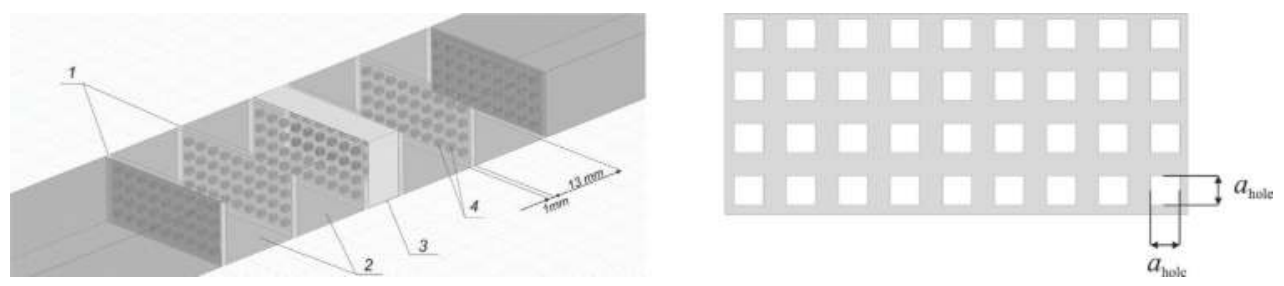

Figure 4. Model of the photonic crystal: 1-layers of ceramics with square through holes, 2-polystyrene layers, 3disturbance in the form of a polystyrene layer with changed thickness (a). The ceramics layer with square through holes (b). 
peak in the forbidden band of the photonic crystal, the position of which is determined by the size of this disturbance.

Measurement of the amplitude-frequency characteristic of the transmittance of the investigated photonic crystal in the $3 \mathrm{~cm}$ wavelength range was carried out using the Agilent PNAL Network Analyzer N5230A. The results of experimental studies of the amplitude-frequency characteristics of the transmittance of a photonic crystal without disturbance and for different thicknesses of the disturbed polystyrene layer $d_{6 \text { foam }}$ are shown in Figure 5 (discrete curves). Comparison of the calculation and the experiment results indicates their good agreement.

The layers of investigated photonic crystals containing a large number of air inclusions can be considered as composite materials, which are dielectric matrices based on ceramics with fillers in the form of air inclusions. It is known that the dielectric properties of composite materials can be characterized by the value of the effective permittivity $\varepsilon_{\text {ef }}$ determined by the dielectric permittivities of the matrix $\varepsilon_{1}$ and filler $\varepsilon_{2}$ and their volume fractions. The results of the investigation on the possibility of describing the amplitude-frequency characteristics of the transmission coefficient of the investigated photonic crystal, using the model of the "effective" medium [20-22], are given in [19]. The photonic crystal consists of alternating homogeneous layers with effective dielectric permittivity and polystyrene. Its amplitude-frequency characteristic was calculated using a wave transfer matrix between regions with different values of the propagation constant of the electromagnetic wave, determined by the effective permittivity of the ceramic layers with air inclusions and the dielectric permittivity of the polystyrene.

To determine the effective permittivity $\varepsilon_{\mathrm{ef}}$ it is necessary to solve the inverse problem [16]. According to the frequency dependences of the transmittance of a photonic crystal consisting of periodically alternating polystyrene and ceramic layers with air inclusions, the inverse problem was solved using the least squares method.

Figure 6 shows the frequency dependences of the power transmission coefficient of the photonic crystal with a different fraction of air inclusion in ceramic layers with the disturbed sixth polystyrene layer of thickness $d_{6 \text { foam }}=6.0 \mathrm{~mm}$ (circles) calculated using the finite element method in the CAD ANSYS HFSS and the frequency dependences of the transmission coefficients of a

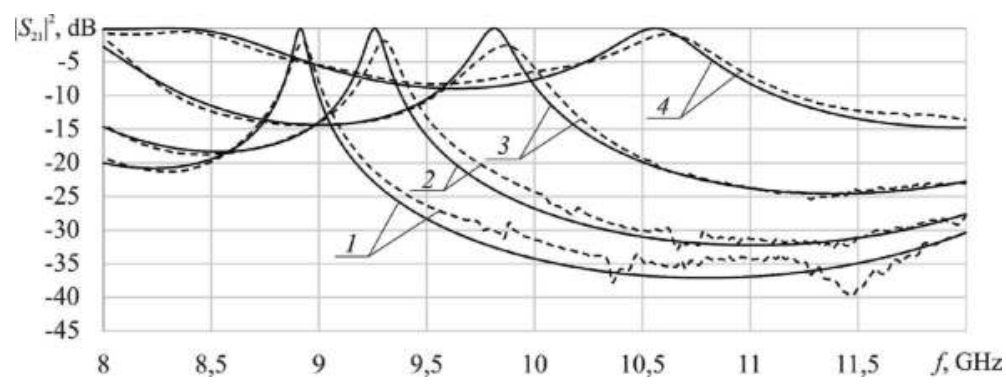

Figure 5. Calculated (continuous curves) and experimental (discrete curves) amplitude-frequency characteristics of the transmittance of a photonic crystal for various volume fractions of air inclusions with disturbed sixth polystyrene layer of $6.0 \mathrm{~mm}$. The volume fraction of inclusions: $1-0,2-8.5,3-23.0$ and $4-43.0 \%$. 
photonic crystal (curves) calculated at the values of the effective permittivity $\varepsilon_{\text {ef }}$ found from the inverse problem solution.

The dependence of the effective permittivity of the layers on the volume fraction of air inclusions, calculated from the solution of the inverse problem, coincides with the dependence described by Maxwell-Garnett ratio [20] with the error of 7.47\%, the Bruggeman ratio [21] with the error of $4.50 \%$ and the Lichteneker ratio [22] with the error of $24.02 \%$.

A distinctive feature of microwave photonic crystals is the ability to provide various functions necessary for the operation of microwave circuits [23-26] with a relatively small number of elements that make up a photonic crystal. A small number of elements forming microwave photonic crystals is associated with the need for compactness of the devices created on their basis. It is of scientific and practical interest to create multielement microwave photonic crystals characterized by small dimensions. To solve this kind of problem, it is possible to use as a photonic crystal the structure that excites higher type waves whose wavelengths are substantially shorter than the wavelength in the waveguide of the main type, as suggested by the authors in [27]. Therefore, the dimensions of devices at higher type waves become significantly smaller than similar devices on the main type of the wave. In this regard, they can be called low dimensional. The results of studies of waveguide photonic crystals in the form of alternating dielectric layers (even elements) of a photonic crystal and thin metal plates (odd elements) partially overlapping the waveguide cross-section are presented in [27]. Between the plates and the wide walls of the waveguide, there were gaps. Each of the plates created gap of the same width along the entire length of the plate. The gaps between the odd metal plates and the waveguide were created at one of the wide walls of the waveguide and the gaps between the even metal plates and the waveguide at the opposite wide wall of the waveguide. In this case, the gap is a source of higher type waves, forming in its vicinity the so-called near field. The structure of the investigated low-dimensional waveguide microwave photonic crystal is shown in Figure 7. The following materials were used as the dielectric material: polystyrene $\left(\varepsilon_{r}=1.02\right)$ and teflon $\left(\varepsilon_{r}=2.1\right)$. The dielectric layers completely filled the cross section $(23 \times 10 \mathrm{~mm})$ of the waveguide. The metal plates $50 \mu \mathrm{m}$ thick were made of aluminum. The width of the gap $S$ did not exceed one-tenth of the size of the waveguide narrow wall.

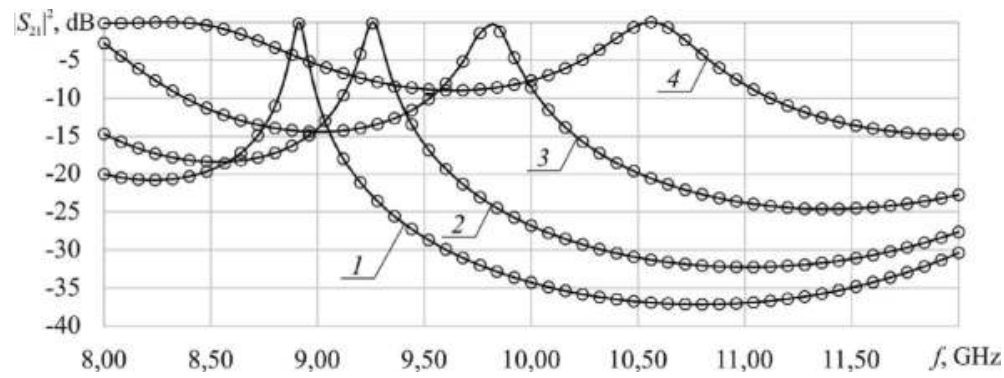

Figure 6. Amplitude-frequency characteristics of the transmission coefficients of a photonic crystal calculated using the values of the effective permittivity $\varepsilon_{\mathrm{ef}}$, found from the inverse problem solution, of ceramics layers with different fraction of air inclusions with the disturbed sixth layer of $6.0 \mathrm{~mm}, \varepsilon_{\text {ef }}$, rel. units: $1-9.6\left(x_{1}=0 \%\right), 2-8.44\left(x_{1}=8.5 \%\right), 3-6.78$ $\left(x_{1}=23.0 \%\right), 4-4.82\left(x_{1}=43.0 \%\right)$. 
Based on numerical simulation, using the finite element method in the CAD ANSYS HFSS, the influence of the thickness of dielectric layers, the gap width and the number of layers of the photonic crystal structure on the amplitude-frequency characteristics of the transmission and reflection coefficients of a photonic crystal was investigated.

The finite-element method was used to calculate the amplitude-frequency characteristic of the transmission $S_{12}$ and the reflection $S_{11}$ coefficients of a nine-layer photonic crystal consisting of five consistently alternating metal plates with gaps and four dielectric layers for different thicknesses of the dielectric layers $h$. It follows from the calculation results that the amplitudefrequency characteristic of the transmittance $S_{12}$ of the structure under study has a "band" character. The amplitude-frequency characteristic of such a photonic crystal consists of specific alternating "allowed" and "forbidden" bands. The frequency position of the resonance peaks of the coefficient $S_{11}$ corresponds to the position of the peaks of the coefficient $S_{12}$. The decrease in the thickness $h$ of the dielectric layers of the structure, as well as the increase in the width of the gap $S$, led to a shift in the amplitude-frequency characteristic of the photonic crystal toward shorter wavelengths and the increase in the width of the "allowed" band. At the same time, the width and depth of the "forbidden" band decreased. It was found that with the number of metal plates in the photonic crystal structure equal to $m$, the number of resonances on its amplitude-frequency characteristic is $m-1$.

Comparison of the results of calculations and experimental studies of a photonic crystal created in accordance with the model described above indicates their good qualitative agreement.

The use of metal plates with gaps in the structure of a photonic crystal has made it possible to substantially reduce its longitudinal dimension to $12.25 \mathrm{~mm}$, which is approximately five times smaller than the longitudinal dimension of a photonic crystal created on elements made of alternating layers of dielectrics with different permittivity.

The disturbance of periodicity in a low-dimensional microwave photonic crystal, as well as in the ordinary one, should lead to the appearance of a defect (impurity) mode resonance. The theoretical definition of the conditions for its appearance and their experimental realization

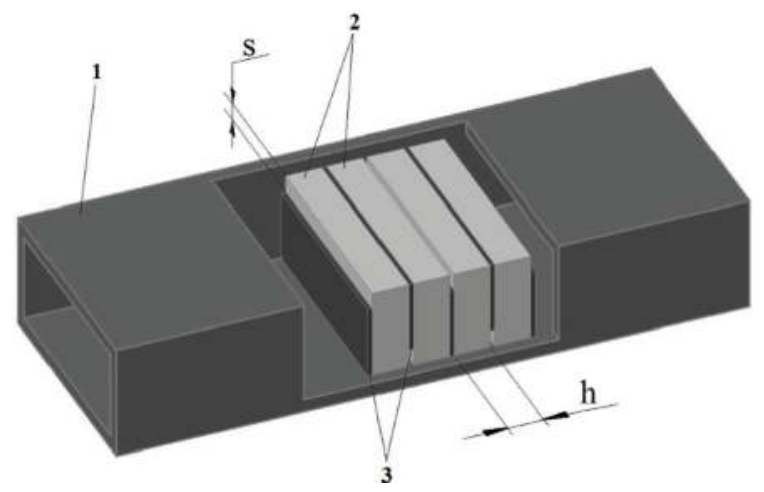

Figure 7. Model of a photonic crystal: 1 -rectangular waveguide segment, 2 -dielectric layers, 3 -thin metal plates, $S$-gap width, $h$-dielectric layer thickness. 
was described in [28]. A violation of the periodicity of a photonic crystal can be a different size of the central dielectric layer or a modified size of the capacitive gap of the diaphragm adjacent to the disturbed layer. In [28], the results of studies of the low-dimensional photonic crystals described above (the inset to Figure 8) are presented. The diaphragms of $50 \mu \mathrm{m}$ thick were made of aluminum. The thickness of each dielectric layer in the photonic crystal without "disturbances" was $3 \mathrm{~mm}$. Thus, the total longitudinal dimension of the crystal without breaking the periodicity was $\sim 15 \mathrm{~mm}$. Figure 8 shows the experimental and calculated using the ANSYS HFSS CAD the transmission coefficient $S_{21}$ of an eleven-layer photonic crystal without disturbance (curves 3,4) and with disturbance (curves 1,2) as a central dielectric (teflon, $\varepsilon_{r}=2.1$ ) layer with thickness reduced to $1 \mathrm{~mm}$, with a fixed value of the gap $S=1 \mathrm{~mm}$.

The measurements were carried out using the Agilent PNA-L Network Analyzer N5230A. Comparison of the calculations and measurements results indicates their qualitative agreement. The existing difference may be due to ignoring the attenuation in the waveguide walls, which is essential for higher types of waves.

As follows from the results shown in Figure 8, the introduction of the disturbance in the form of a changed size of the central dielectric layer led to the appearance of a defect mode resonance and a significant change in the width and depth of the "forbidden" band. At the same time, the position of the defect mode on the frequency scale essentially depends not only on the thickness of the "disturbed" dielectric layer but also on the value of the capacitive gap of the diaphragms.

Periodic structures based on resonators as retarding systems for vacuum microwave devices and microwave filters have been described as early as in the 1960s [28, 29]. They were intended

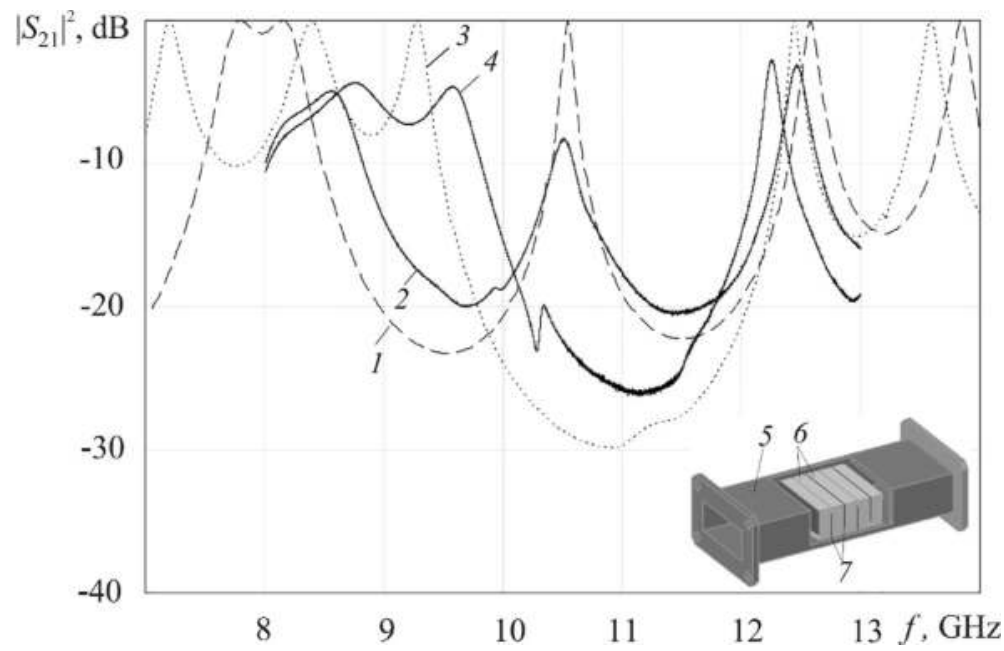

Figure 8. Calculated (dashed and doted curves) and experimental (solid curves) amplitude-frequency characteristics of transmittance $S_{12}$ of 11-layer low-dimensional photonic crystal: curves 1,2-with "disturbed" sixth central teflon layer of $1 \mathrm{~mm}$; curves 3 and 4 -without the periodicity disturbance. On the inset: a photonic crystal model: 5-rectangular waveguide segment, 6-dielectric layers, 7-capacitive diaphragms. 
to be used as delay-line structures in these devices, providing the optimal interaction of the electron beam with the electromagnetic wave. The investigated structure consists of periodically located metal resonant diaphragms at a distance $l$ from each other, deposited to a dielectric substrate (Figure 9) [30].

On the basis of numerical simulation using the finite element method in the CAD ANSYS HFSS, the influence of substrates with different dielectric permittivity on the microwave reflection and transmission coefficients of the structure was investigated. The amplitudefrequency characteristics of a photonic crystal made up of metal diaphragms deposited on a dielectric substrate with a through aperture for different widths of the aperture and the amplitude-frequency characteristics of a photonic crystal made up of diaphragms on dielectric substrates with apertures filled with a material with the permittivity $\varepsilon_{2}$ were analyzed. The increase in the width of the aperture, for a fixed permittivity of the substrate, causes the increase in the width and depth of the band gap. Moreover, the low-frequency edge of the band remains stationary around $9 \mathrm{GHz}$, and the extension occurs due to the shift from the high-frequency edge of the forbidden band to the high-frequency region. The same tendency is observed when the permittivity of insulator within the aperture increases.

The photonic crystal based on diaphragms without dielectric substrates consisted of 6 aluminum diaphragms of $10 \mu \mathrm{m}$ fixed between two layers of $2 \mathrm{~mm}$ polystyrene placed in a rectangular waveguide. The width and height of the apertures of the diaphragms of the photonic crystal were chosen equal to 14 and $1 \mathrm{~mm}$, respectively. This provided the appearance on the frequency dependences of the transmission and reflection coefficients (the dashed curves in Figure 10a and $\mathbf{b}$, respectively) in the frequency range $8-12 \mathrm{GHz}$ of one allowed and one forbidden bands. The same figures show the results of measurements of the amplitudefrequency characteristics of a photonic crystal with a disturbance of periodicity (solid curves) in the form of a modified distance $L$ between the central diaphragms.

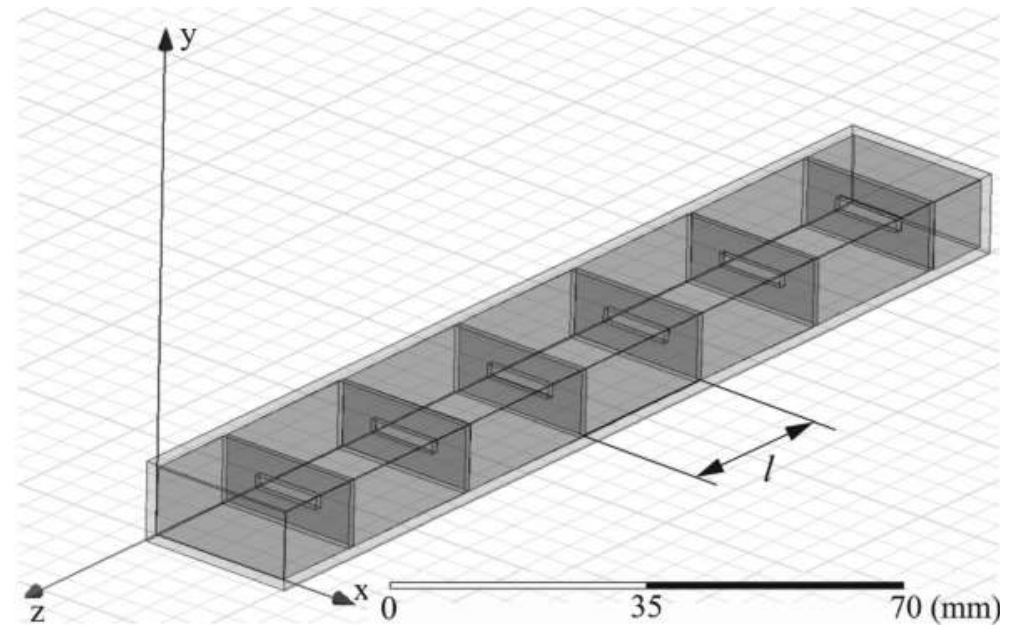

Figure 9. The scheme of the microwave photonic crystal, where $l$ is the distance between the diaphragms. 

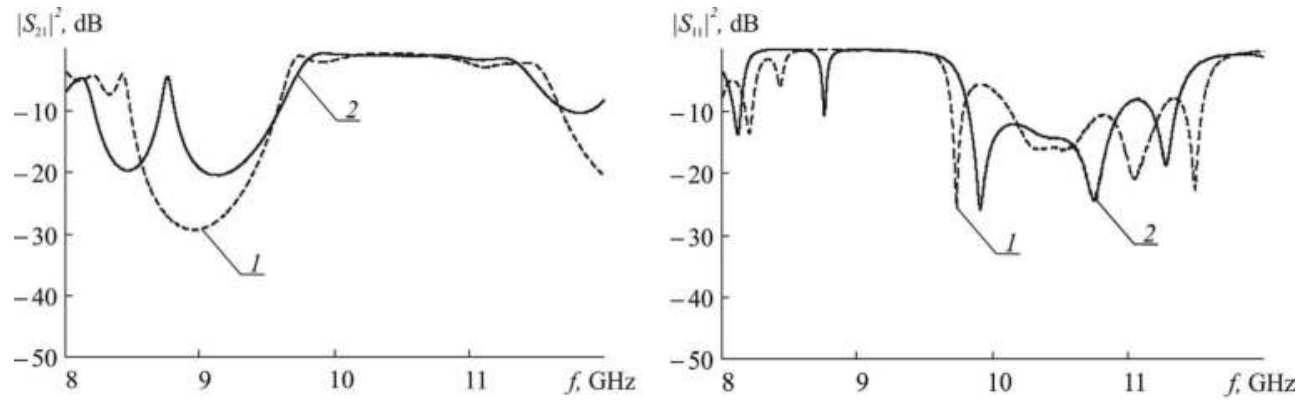

Figure 10. Experimental amplitude-frequency characteristics of the transmission coefficient (a) and reflection (b) of a photonic crystal based on diaphragms without dielectric substrates in the case of the disturbance absence (curve 1) and the disturbance presence (curve 2). Distance between diaphragms $l=27 \mathrm{~mm}$, length of slots $a_{1}=14 \mathrm{~mm}$, size of disturbance in the central layer $L=20 \mathrm{~mm}$.

A comparison of the experimental dependences presented in Figure 10 with the results of the calculation of the amplitude-frequency characteristics of the photonic crystal based on diaphragms without dielectric substrates in the case of the disturbance presence and the disturbance absence with parameters corresponding to the experimental sample described above indicates good qualitative and quantitative agreement.

\subsection{Control of the characteristics of microwave photonic crystals}

The ability to control the amplitude-frequency characteristics of microwave photonic crystals opens the prospect of expanding their field of application. This possibility was considered, in particular, in [31]. The authors of [31] took the design of the microwave photonic crystal used in [32] as a filter. Polystyrene, ceramics $\mathrm{Al}_{2} \mathrm{O}_{3}$ (thickness of $1 \mathrm{~mm}$ ) and polycrystalline yttriumiron garnet (YIG) (thickness of $1 \mathrm{~mm}$ ) were used as the material forming the photonic crystal layer. The control of the amplitude-frequency characteristics of the investigated structures by magnetic field is provided by the increase in the real part of the ferrite magnetic susceptibility under increasing magnetic field. This leads to the increase in the microwave field concentration in the ferrite and to the increase in the phase shift of the wave as it passes through the ferrite plate and, as a consequence, to the shift of the amplitude-frequency characteristic.

In [33], the possibility of creating the waveguide photonic crystal with a tunable frequency position of a transparency window associated with the periodicity disturbance in the photonic crystal and with attenuation in this window controlled by $p-i-n$ diodes was shown. The 11layer microwave photonic crystal designed for operation in a 3-cm wavelength range was created, consisting of 11 alternating layers of ceramics $\mathrm{Al}_{2} \mathrm{O}_{3}\left(\varepsilon_{r}=9.6\right.$, thickness of $\left.1 \mathrm{~mm}\right)$ and polystyrene $\left(\varepsilon_{r}=1.1\right.$, thickness of $\left.1 \mathrm{~mm}\right)$. The disturbance of periodicity was provided by using polystyrene plate as the sixth layer. To provide control of the transmittance in the transparency band the $p$-i-n-diode array was used, which was placed into the waveguide in conjunction with the photonic crystal (Figure 11). 


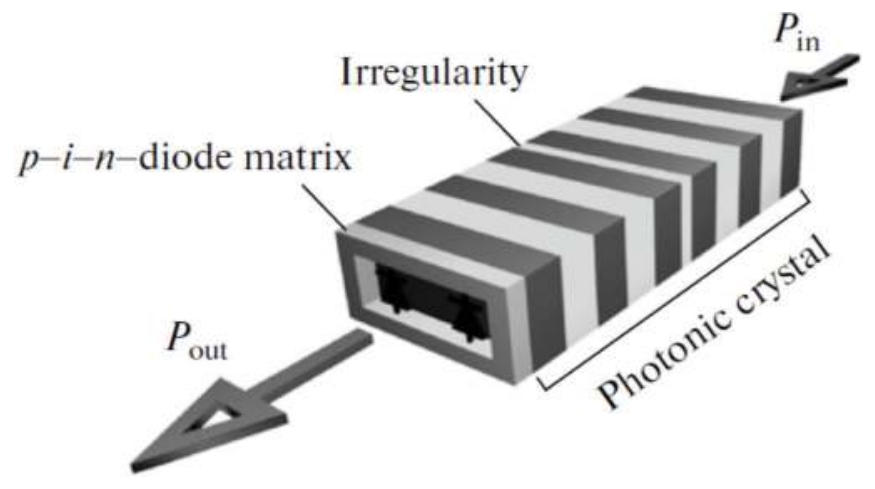

Figure 11. The arrangement of the photonic crystal and the $p$-i-n-diode array.

The control voltage regulated in the range $0-700 \mathrm{mV}$ was applied to the $p$ - $i$ - $n$-diodes matrix. The matrix of $p$-i-n-diodes, in the absence of bias voltage, introduces a weak perturbation into the photonic crystal, and its characteristics remain practically unchanged. With increasing bias voltage, this perturbation increases due to the enrichment of the $i$-region by the charge carriers, and specificity for the photonic crystal resonant transmission decreases. The experimental frequency dependences $\left|S_{12}\right|^{2}, \arg S_{12}$ and $\left|S_{11}\right|^{2}, \arg S_{11}$ are shown in Figure 12 for different bias voltage values on the $p-i$-n-diode with the thickness of the disturbed layer $d_{6}=5 \mathrm{~mm}$. As it follows from the results in this figure, the use of the microwave photonic crystal makes it possible to create the microwave switch with electrically adjustable characteristics from -1.5 to $-25 \mathrm{~dB}$ when the bias voltage on $p-i-n$ diodes varies from 0 to $700 \mathrm{mV}$.

It is known that the ring-type structures demonstrate properties of a photonic crystal, such as the presence of forbidden and allowed bands. The band nature of the spectrum in such structures is provided due to multiple reflections from the inhomogeneity in the structure. Such devices in microstrip design are given in [34,35]. The characteristics of such structures in waveguide design are given in [36]. The element of the type "metal pin with a gap" is used in them as inhomogeneity. This inhomogeneity provides the appearance of a resonant feature in the forbidden band of the system under investigation, called the defect mode resonance or "transparency window." Accordingly, the blocking peak may appear in the allowed band (passband). Figure 13 shows the design of a microwave element based on diaphragm, the system of coupled frame elements with "pin with a gap" type inhomogeneity located $20 \mathrm{~mm}$ to the right of the diaphragm and the semiconductor $n-i-p-i-n$-structure located in the gap between the pin and the frame element.

As it follows from the calculation results, the change in the conductivity of the control element from $10^{-3}$ to $10^{5} \mathrm{~S} / \mathrm{m}$ leads to a change in the transmission coefficient at $9.44 \mathrm{GHz}$, corresponding to the blocking peak, in the range -36.79 to $-1.01 \mathrm{~dB}$. The system whose construction was described above was investigated experimentally. The bias voltage applied to the $n-i-p-i-n$ structure in the $0-9 \mathrm{~V}$ range resulted in the change of the transmission coefficient from -25 to $-1.5 \mathrm{~dB}$ at $9.644 \mathrm{GHz}$. 


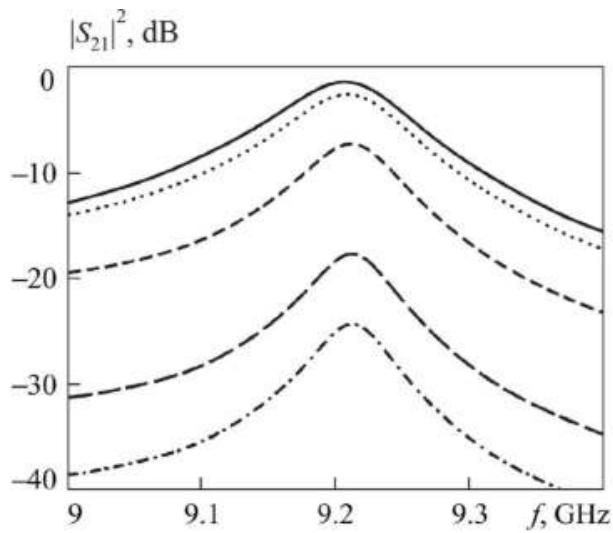

$a$

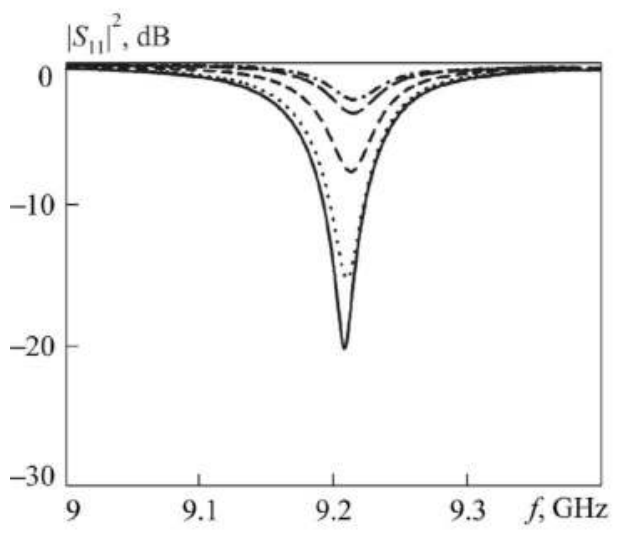

c
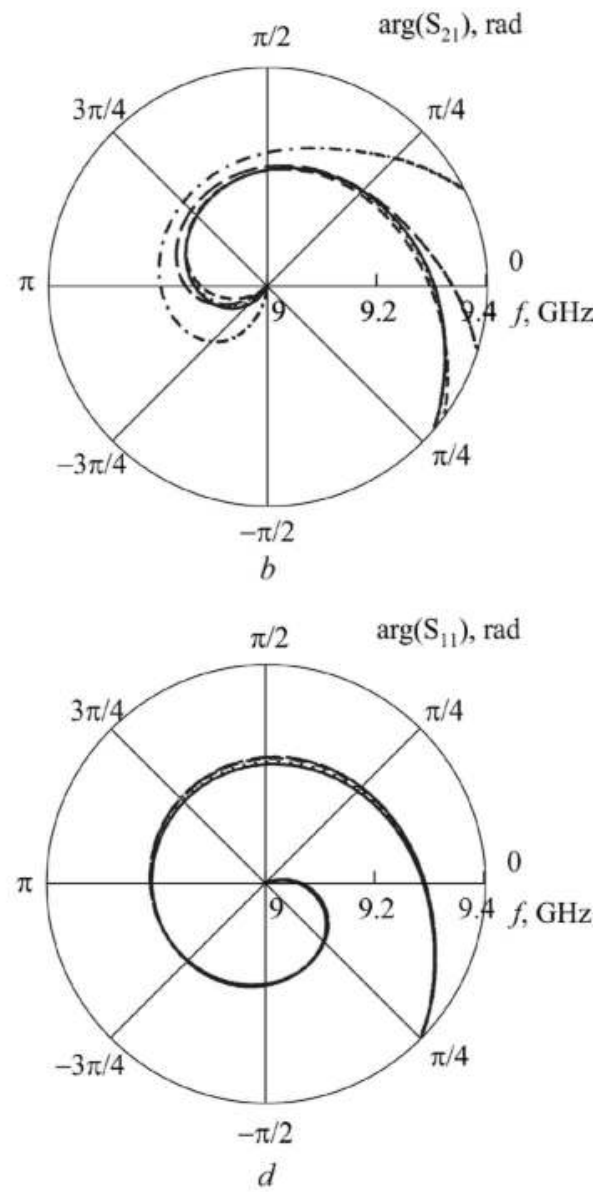

$\longrightarrow U=0 \cdots \cdots \cdot U=400 \mathrm{mV}----U=500 \mathrm{mV}--\cdot U=600 \mathrm{mV}-\cdot-\cdot-U=700 \mathrm{mV}$

Figure 12. Experimental dependences of the square of the modulus $(a, b)$ and phase $(b, d)$ of the transmission $(a, b)$ and the reflection $(\mathrm{c}, \mathrm{d})$ coefficients of electromagnetic radiation in the region of the transparency window of a photonic crystal for different values of the voltage on the $p$-i-n-diode, $d_{6}=5.5 \mathrm{~mm}$.

\subsection{Application of microwave photonic crystals}

Different directions of practical use of the unique characteristics of microwave photonic crystals were considered in [25]. One of the common names of microwave photonic crystals is structures with a forbidden band. At the same time, it is known that, in addition to the forbidden band (an analog of the band gap in semiconductors), microwave photonic crystals demonstrated the frequency band in which the wave propagates, practically without attenuation. The authors of [37] proposed to use this property to create waveguide broadband 


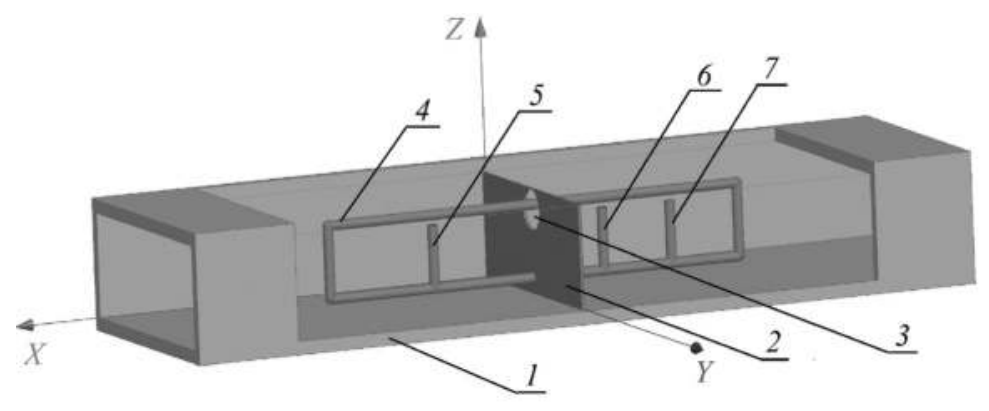

Figure 13. Model of the microwave element based on the diaphragm and the system of coupled frame elements containing inhomogeneity in the form of "pin with a gap" designs: 1-waveguide, 2-diaphragm, 3-aperture, 4-frame element, 5-7-inhomogeneities of "pin with a gap" type.

matched loads. The problem of constructing such loads remains one of the topical problems in microwave radioelectronics at the present time. Matched microwave loads are widely used both independently and as elements of complex functional devices: directional couplers, summators, power meters, measuring bridges, microwave filters, and so on [38, 39].

The results of studies of frequency dependences of the reflection coefficients of electromagnetic radiation from one-dimensional photonic crystals containing, along with periodically changing dielectric filling, nanometer metal layers are presented in $[9,10]$. Computer simulations were carried to determine the possibility of using one-dimensional photonic crystals for creating matched loads. When calculating the reflection $S_{11}$ and the propagation $S_{21}$ coefficients of an electromagnetic wave, the matrix of wave transfer between regions with different values of the propagation constant of the electromagnetic wave was used, as was described in detail in $[10,16,17]$. During computer simulation in [2], the possibility of creating waveguide matched loads in an 8- and 3-cm wavelength bands was demonstrated.

The authors of [40] showed, as a result of solving the optimization problem, including the choice of the surface resistance of a nanometer metal film, that it was possible to create the matched load of the described above type with the voltage standing wave ratio (VSWR) not more than 1.1 in the frequency bands $8.15-12.05,12.05-17.44$ and $17.44-25.95 \mathrm{GHz}$. The longitudinal dimensions of the loads were less than 14.5, 10.0, $9.0 \mathrm{~mm}$, respectively. For the frequency range 25.95-37.50 GHz (Figure 14), the VSWR of the created load was less than 1.15 with a longitudinal dimension of $6.5 \mathrm{~mm}$.

It is known that reflection and transmission spectra of electromagnetic radiation can be used to measure the thickness and electrical conductivity of a semiconductor in metal-semiconductor structures [15-17]. The sensitivity of these methods depends significantly on how much these spectra are changed under changing the values of the semiconductor parameters. The determination of these parameters from the results of measurements of spectral dependences is called the solution of the inverse problem. The authors of [41] proposed to use for measurements of the parameters of nanometer metal layers on insulating substrates waveguide photonic structures in 


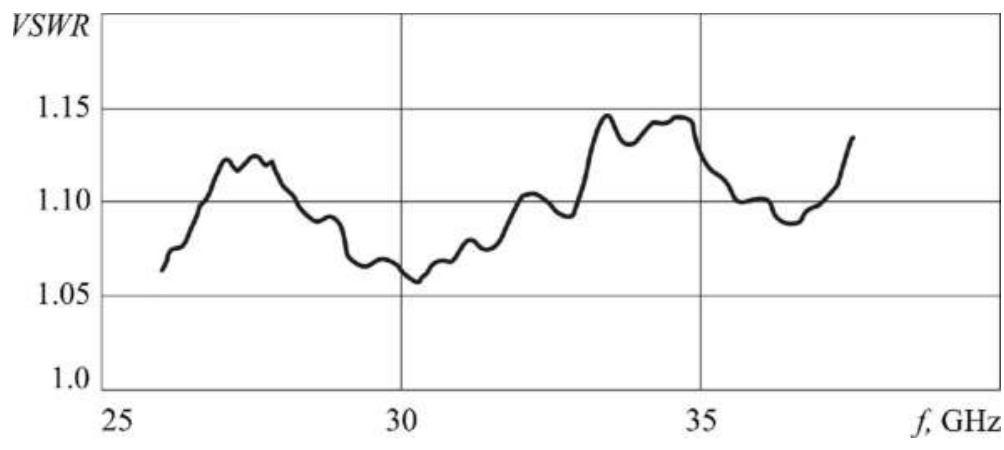

Figure 14. Experimental frequency dependence of VSWR in the range 25.95-37.50 GHz.

which the metal-dielectric structure was placed in a segment of a microwave photonic crystal that disturbed its periodicity. In the "forbidden" band of the photonic crystal, the transparency "window," that is sensitive to the sought parameters of the metal-dielectric structure, is seen.

One of the most successful and promising methods for diagnosing materials and structures that allow measurements with high spatial resolution is near-field microwave microscopy [42-44]. A key element of the near-field microwave microscope is a probe with an aperture size much smaller than the wavelength of the microwave radiation. In near-field microwave microscopes, the field of a non-propagating wave type is used as a probing source [16, 42, 43]. This field that is formed if the central conductor of the coaxial line goes beyond the outer conductor [15]. The authors [13] called the microwave resonator connected to the probe as the main element of the near-field microwave microscope, which provides to a greater extent its high sensitivity and resolution.

The increase in the sensitivity of the resonator to the perturbation introduced into it through the probe causes the increase in the sensitivity and resolution of the microwave microscope as a whole.

The design of the resonator in conjunction with the probe element for a near-field microwave microscope is described in [45]. In [46], the results of studying the possibility of using the onedimensional photonic crystal in which a tunable resonator, serving as a load, allows to control the resonance features in the reflection spectrum of a near-field microwave microscope probe were presented. As such a load, the authors of [46] chose the cylindrical resonator with the coupling element extending beyond the cavity, the end of which is used as a probe of a nearfield microscope to monitor the parameters of the dielectric plate with different values of the permittivity and the thickness of nanometer metal layers deposited on dielectric plates.

The general view of the probe of a near-field microwave microscope on the basis of a cylindrical microwave resonator with the coupling frame element and the one-dimensional photonic crystal is shown in Figure 15.

The probe based on the cylindrical resonator with a coupling frame element was connected to a segment of the waveguide photonic crystal 9 with the disturbance of the periodicity. The 


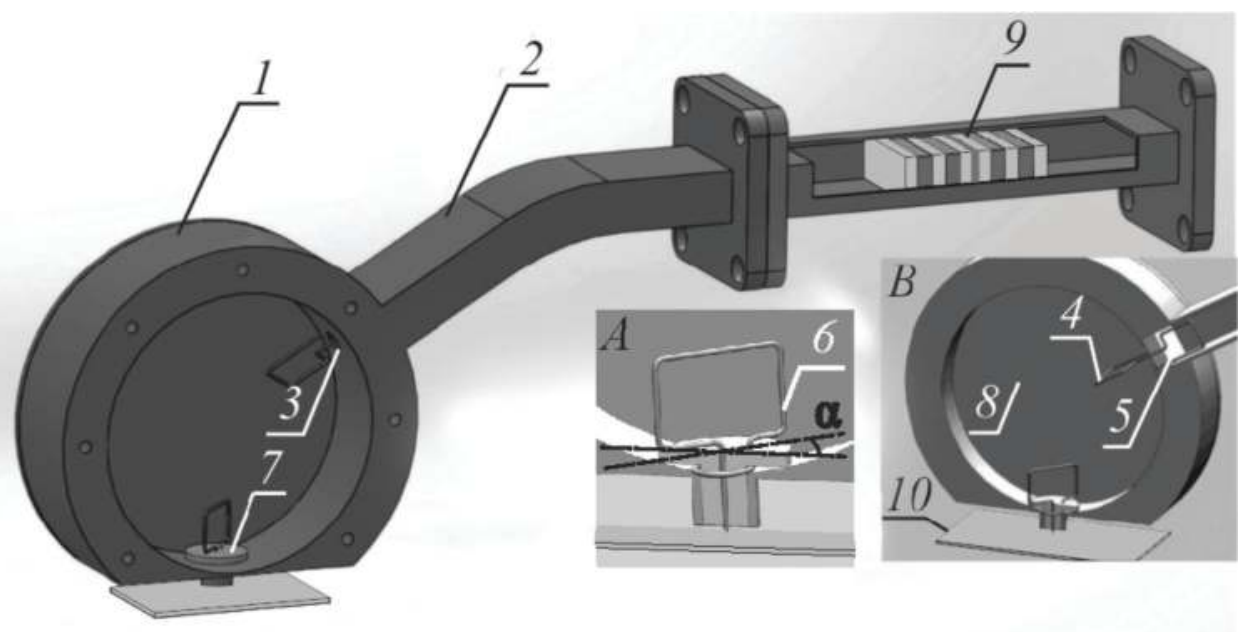

Figure 15. Probe of a near-field microwave microscope based on a cylindrical microwave cavity 1 with a communication coupler 6 and a one-dimensional photonic crystal 9. Insert A is the frame coupling element. Insert B is the cylindrical microwave resonator with the coupling frame element and the measured sample 10.

one-dimensional waveguide photonic crystal consisting of 11 layers was used in the frequency range 8-12 GHz. The odd layers were made of ceramics $\mathrm{Al}_{2} \mathrm{O}_{3}\left(\varepsilon_{r}=9.6\right)$, even layers were made of teflon $\left(\varepsilon_{r}=2.1\right)$. The length of odd segments was $1 \mathrm{~mm}$, of even segments varied in the range from 7 to $14 \mathrm{~mm}$. The disturbance of periodicity was created by changing the length of the sixth central layer. The length of the disturbed sixth (teflon) layer varied in the range from 3 to $4 \mathrm{~mm}$.

The tip of the probe approaching to the sample caused abrupt change in the input impedance of the probe and the reflection coefficient of the microwave wave from the probe. The magnitude of its change depends on the parameters of the test sample, such as electrical conductivity, permittivity, thickness.

Figure 16 shows the results of measurements of the frequency dependence of the microwave reflection coefficient in the vicinity of the resonant frequency at a fixed gap $(18 \mu \mathrm{m})$ between the probe and the samples under study with different dielectric permittivity. Figure $\mathbf{1 6}$ shows the dependences of the microwave reflection coefficient measured at various fixed frequencies in the vicinity of the reflection coefficient minimum on the dielectric permittivity of the samples placed at a fixed distance near the tip of the probe.

The investigated resonance system can also be used to measure samples in the form of dielectric plates with nanometer metal layers of different thicknesses. Figure 17 shows the results of measurements of the frequency dependences of the microwave reflection coefficient in the vicinity of the resonance frequency at a fixed gap $(18 \mu \mathrm{m})$ between the probe and the samples under study with different thicknesses of the nanometer metal layer $(\mathrm{Cr})$ in the metalinsulator structure. The thickness of the deposited nanometer metal layers was measured using the Agilent Technologies AFM5600 atomic force microscope. 


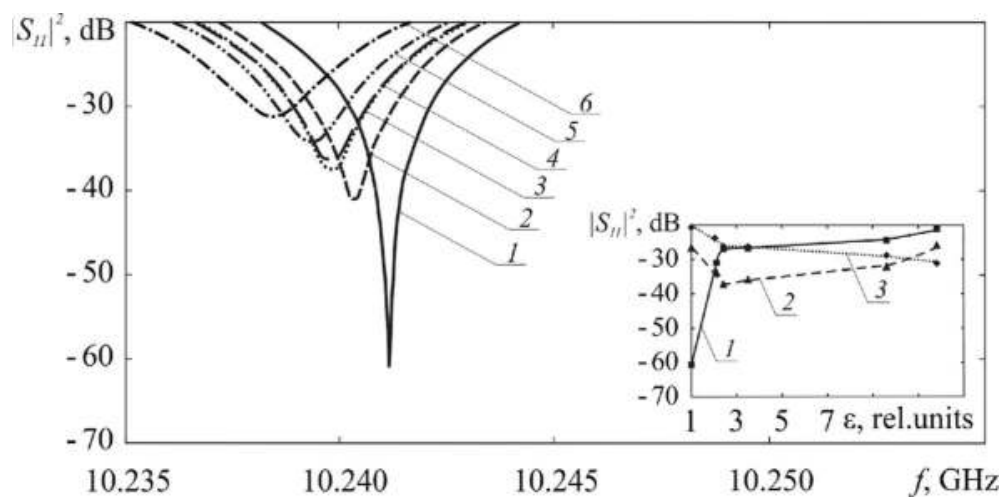

Figure 16. Frequency dependences of the microwave reflection coefficient in the vicinity of the resonance frequency at a fixed gap equal to $18 \mu \mathrm{m}$ between the probe and samples with different dielectric permittivity. Curve 1 corresponds to the absence of the measured sample $\left(\varepsilon_{r}=1\right), 2$-teflon $\left(\varepsilon_{r}=2.0\right), 3$-paper-based laminate $\left(\varepsilon_{r}=2.5\right), 4$-textolite; $\left(\varepsilon_{r}=3.4\right)$, 5-ceramics $\mathrm{Al}_{2} \mathrm{O}_{3}\left(\varepsilon_{r}=9.6\right), 6$ - silicon $\left(\varepsilon_{r}=11.7\right)$.

The inset of Figure 17 shows the dependences of the microwave reflection coefficient measured at various fixed frequencies $(10.77908,10.77895$ and $10.77850 \mathrm{GHz})$ in the vicinity of the reflection coefficient minimum on the thickness of the nanometer metal layer on ceramics $\mathrm{Al}_{2} \mathrm{O}_{3}$ plates placed at a fixed distance near the tip of the probe.

In [47], it is proposed to implement a method for simultaneous measurement of the substrate thickness of a semiconductor structure, the thickness and conductivity of a heavily doped epitaxial layer, the mobility of free charge carriers in this layer using a one-dimensional microwave photonic crystal. The one-dimensional waveguide photonic crystal consisted of 11 layers, forming a structure of periodically alternating elements, each of which included two

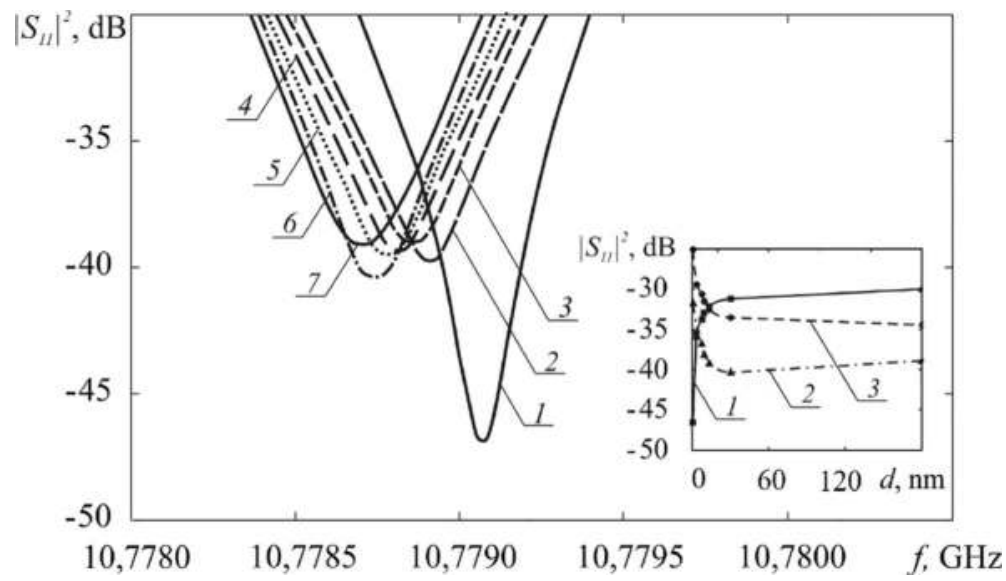

Figure 17. Frequency dependences of the microwave reflection coefficient in the vicinity of the resonance frequency at a fixed gap equal to $18 \mu \mathrm{m}$ between the probe and the samples under study with different thicknesses of the nanometer metal layer Cr: (1) without metallization, (2) $d=3 \mathrm{~nm},(3) d=7 \mathrm{~nm}$, (4) $d=9 \mathrm{~nm}$, (5) $d=13 \mathrm{~nm}$, (6) $d=30 \mathrm{~nm}$, and (7) $d=180 \mathrm{~nm}$. 
layers, was considered. The measured structure, placed on the boundary of the disturbed central teflon layer and the next ceramics $\mathrm{Al}_{2} \mathrm{O}_{3}$ layer, was oriented in two ways relative to the direction of the electromagnetic wave propagation. The location of the sample inside the disturbed layer and its orientation relative to the disturbed layer in the photonic crystal are shown in Figure 18 (configuration 1 and 2).

The investigated samples were epitaxial arsenide-gallium structures of thickness $t_{s}=t+t_{\text {sub }}$ consisting of an epitaxial layer with a thickness $t$ and electrical conductivity $\sigma$ and a semiinsulating substrate with thickness $t_{\text {sub }}$. The sought values of the investigated sample parameters are determined by the numerical method as a result of solving the system of equations:

$$
\frac{\partial S\left(t_{\mathrm{sub}}, t, \sigma\right)}{\partial t}=0 ; \frac{\partial S\left(t_{\mathrm{sub}}, t, \sigma\right)}{\partial t_{\mathrm{sub}}}=0 ; \frac{\partial S\left(t_{\mathrm{sub}}, t, \sigma\right)}{\partial \sigma}=0
$$

The residual function $S\left(t_{\mathrm{sub}}, t, \sigma\right)$ defined by expression.

$$
S\left(t_{\text {sub }}, t, \sigma\right)=\sum_{i=1}^{K}\left(\begin{array}{l}
\left(\left|S_{211}\left(\omega_{i}, t_{\text {sub }}, t, \sigma\right)\right|^{2}-\left|S_{21 i 1} \exp \right|^{2}\right)^{2}+\left(\left|S_{111}\left(\omega_{i}, t_{\text {sub }}, t, \sigma\right)\right|^{2}-\left|S_{11 i 1 \exp }\right|^{2}\right)^{2}+ \\
+\left(\left|S_{212}\left(\omega_{i}, t_{\text {sub }}, t, \sigma\right)\right|^{2}-\left|S_{21 i 2 \exp }\right|^{2}\right)^{2}+\left(\left|S_{112}\left(\omega_{i}, t_{\text {sub }}, t, \sigma\right)\right|^{2}-\left|S_{11 i 2 \exp }\right|^{2}\right)^{2}
\end{array}\right),
$$

for the case when the thickness of the heavily doped GaAs layer was $13.14 \mu \mathrm{m}$, its electrical conductivity $\sigma=71.73 \mathrm{Om}^{-1} \mathrm{~m}^{-1}$, and the thickness of the substrate $t_{\text {sub }}=480.3 \mu \mathrm{m}$, has a

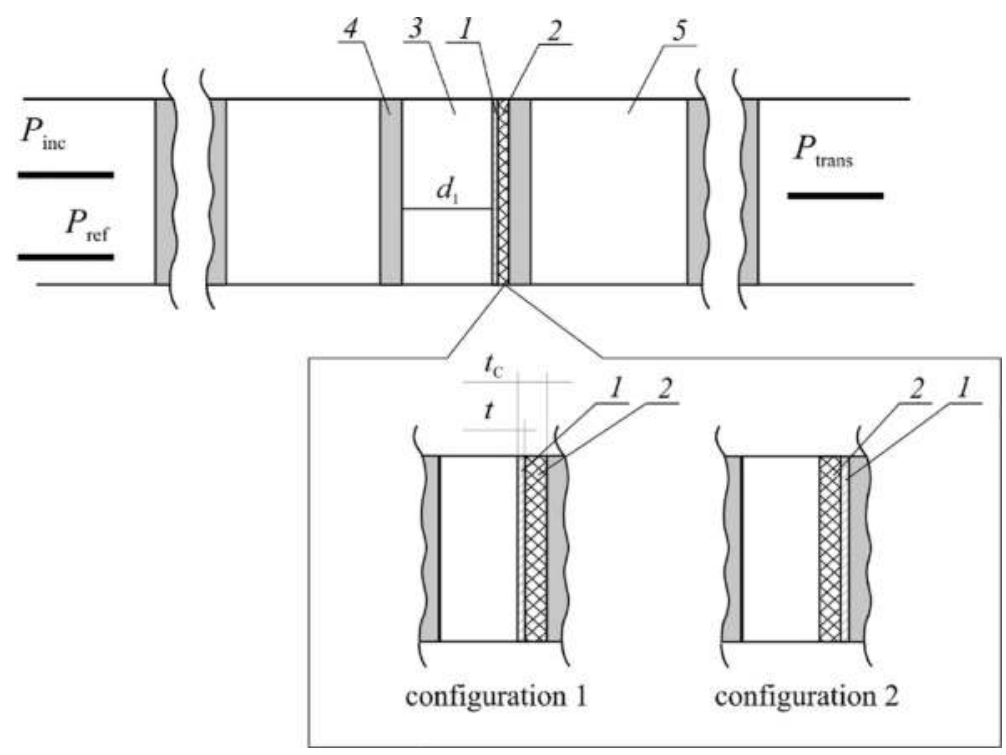

Figure 18. Arrangement of the semiconductor structure relative to the disturbed layer in the waveguide microwave 8 photonic crystal: (1) heavily doped semiconductor layer, (2) high-resistance substrate, (3) disturbed central layer, (4) and (5) periodically alternating layers with different values of permittivity. 


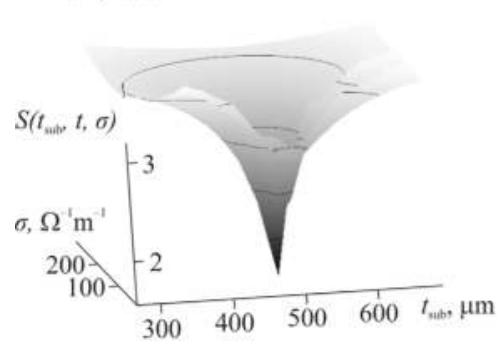

a)

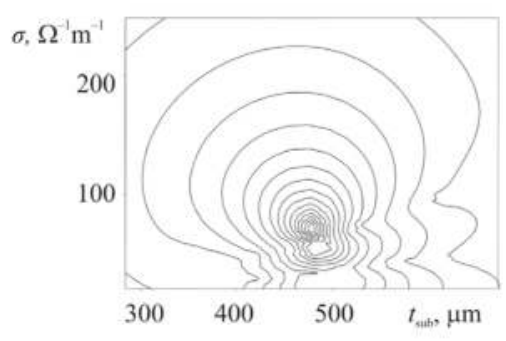

b)

Figure 19. The form of the residual function in space and the contour maps in the planes of the sought parameters $a), b$ ) $-\left(t_{\mathrm{sub}}, \sigma\right)$ for the gallium arsenide sample with an epitaxial layer of thickness $t=13.14 \mu \mathrm{m}$ and electrical conductivity $\sigma=71.73 \mathrm{Om}^{-1} \mathrm{~m}^{-1}$ grown on a high-resistivity substrate of thickness $t_{\text {sub }}=480.3 \mu \mathrm{m}$.

global minimum in the coordinate space $\left(t_{\mathrm{sub}}, t, \sigma, S(t, \sigma)\right)$, and the contour maps (Figure 19) are characterized by the presence of closed trajectories near the minimum. This fact determines possibility to obtain the thickness and electrical conductivity of the semiconductor layer from the solution of the system of equations (Eq. (9)).

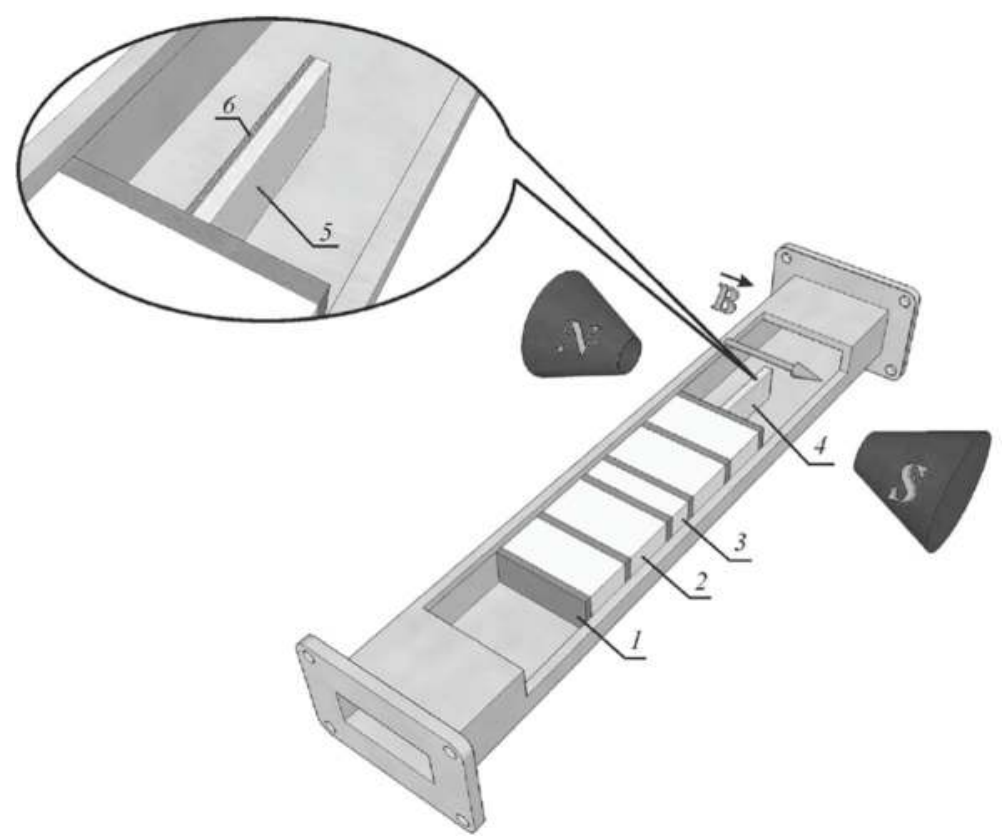

Figure 20. Arrangement of the photonic crystal and epitaxial semiconductor structure in the waveguide. 1-ceramics $\mathrm{Al}_{2} \mathrm{O}_{3}$ layer, 2-teflon layer, 3-disturbed teflon layer, 4-tested gallium arsenide structure, which includes: 5-highresistance substrate, 6 -heavily doped semiconductor layer. $N$ and $S$ are poles of the electromagnet. 
To determine the mobility of free charge carriers of semiconductor heavily doped layers, the investigated epitaxial semiconductor structure was placed in the E plane at the center of the cross section of a rectangular waveguide after a waveguide photonic crystal. The magnetic induction vector of the magnetic field $\vec{B}$ was directed perpendicular to the narrow walls of the waveguide (Figure 20).

To find the mobility of free charge carriers by frequency dependences $S_{21}(\omega)$ and $S_{11}(\omega)$, the least squares method was used, in which the mobility value $\mu$ corresponding to the minimum value of the sum of the squares of the differences between the calculated $\left|S_{21}(\omega, \mu)\right|^{2}$ and $\left|S_{11}(\omega, \mu)\right|^{2}$ and the experimental $\left|S_{21_{\text {exp }}}\right|^{2}$ and $\left|S_{11 e_{x p}}\right|^{2}$ values of the squares of the modules of the transmission and reflection coefficients measured under the influence of the magnetic field and without it. The sought mobility values for two measured structures with thicknesses of heavily doped semiconductor layers of $t=2.17$ and $13.14 \mu \mathrm{m}$ were determined numerically and amounted to $0.591 \mathrm{~m}^{2} /\left(\mathrm{V}^{*} \mathrm{~s}\right)$ and $0.72 \mathrm{~m}^{2} /\left(\mathrm{V}^{*} \mathrm{~s}\right)$, respectively.

\section{Conclusions}

This chapter presents the results of theoretical and experimental studies of one-dimensional microwave photonic crystals based on rectangular waveguides characterized by the presence of forbidden and allowed bands for the propagation of electromagnetic waves. Methods for describing the electrodynamic characteristics of photonic crystals and their relationship with the parameters of periodic structures filling the waveguides have been presented. The change in the width of the allowed and forbidden bands of a photonic crystal has been described when a defect mode occurs because of the creation of the disturbance of the periodicity of the photonic crystal structure. The change in the amplitude-frequency characteristics of microwave photonic crystals has been considered. The types of disturbances and defect modes have been described. The results of the investigation on the characteristics of waveguide microwave photonic crystals created in the form of dielectric matrices with air inclusions have been presented. It has been shown that the layers of the investigated photonic crystals containing a large number of air inclusions can be considered as composite materials. New types of lowdimensional microwave waveguide photonic crystals containing periodically alternating elements that are the source of higher type waves have been described. The possibility of effective control of the amplitude-frequency characteristics of microwave photonic crystals by electric and magnetic fields has been analyzed. The examples of applications of waveguide microwave photonic crystals such as methods for measuring the parameters of materials and semiconductor nanostructures that play the role of periodicity disturbance of the microwave photonic crystals; resonators of near-field microwave microscopes; small-sized broadband matched loads of centimeter and millimeter wavelengths ranges based on microwave photonic crystals have been given. 


\section{Acknowledgements}

The research was carried out with the financial support of the Ministry of Education and Science of the Russian Federation (state task №8.7628.2017/BCH).

\section{Author details}

Dmitry Usanov and Alexander Skripal *

*Address all correspondence to: skripala_v@info.sgu.ru

Saratov State University, Russia

\section{References}

[1] Keldysh LV. The influence of ultrasound on the electronic spectrum of a crystal. Physics of the Solid State. 1962;8:2265-2267

[2] Esaki L, Tsu R. Superlattice and negative differential conductivity in semiconductors. IBM Journal of Research and Development. 1970;14:61-65. DOI: 10.1147/rd.141.0061

[3] Joannopulos J, Villeneuve PR, Fan S. Photonic crystals: Putting a new twist on light. Nature. 1997;386:143-146. DOI: 10.1038/386143a0

[4] Yablonovitch E. Inhibited spontaneous emission in solid-state physics and electronics. Physical Review Letters. 1987;58:2059-2063. DOI: 10.1103/PhysRevLett.58.2059

[5] John S. Strong localisation of fotons in certain disordered dielectric superlattices. Physical Review Letters. 1987;58:2486-2491. DOI: 10.1103/PhysRevLett.58.2486

[6] Zaitsev DF. Nanophotonics and Its Application. Moscow: Acteon; 2012. 445p

[7] Silin RA, Sazonov VP. Delay-Line Systems. Moscow: Soviet Radio; 1966. 628p

[8] Tae-Yeoul, Chang K. Uniplanar one-dimensional photonic-bandgap structures and resonators. IEEE Transactions on Microwave Theory and Techniques. 2001;49:549-553. DOI: $10.1109 / 22.910561$

[9] Usanov D, Skripal Al, Abramov A, Bogolyubov A, Skvortsov V, Merdanov M. Measurement of the metal nanometer layer parameters on dielectric substrates using photonic crystals based on the waveguide structures with controlled irregularity in the microwave band. In: Proceedings of 37th European Microwave Conference; 8-12 October 2007; Munich, Germany. pp. 198-201. DOI: 10.1109/EUMC.2007.4405160 
[10] Gulyaev YV, Nikitov SA. Photonic and magnetophotonic crystals-A new medium for information transfer. Radioengineering. 2003;8:26-30

[11] Samoilovich MI, Tsvetkov MY. Rare earth opal nanocomposites for nanophotonics. Nanoand Microsystem Technology. 2006;10:8-14

[12] Kul'chin YN, Bagaev SN, Bukin OA, Voznesenskiı̌ SS, Drozdov AL, Zinin YA, Nagorny IG, Pestryakov EV, Trunov VI. Photonic crystals based on natural oceanic biominerals. Technical Physics Letters. 2008;8:633-635. DOI: 10.1134/S1063785008080014

[13] Usanov DA, Rytik AP. Properties of a photonic crystal formed in a solution featuring the Briggs-Rauscher oscillating reaction. Technical Physics Letters. 2016;42:629-631. DOI: 10.1134/ S1063785016060304

[14] Gomez A, Vegas A, Solano MA, Lakhtakia A. On one- and two-dimensional electromagnetic band gap structures in rectangular waveguides at microwave frequencies. Electromagnetics. 2005;25:437-460. DOI: 10.1080/02726340590957443

[15] Usanov DA, Skripa AV, Abramov AV, Bogolyubov AS. Determination of the metal nanometer layer thickness and semiconductor conductivity in metal-semiconductor structures from electromagnetic reflection and transmission spectra. Technical Physics. 2006;5:644-649. DOI: $10.1134 / S 1063784206050173$

[16] Chaplygin YA, Usanov DA, Skripal AV, Abramov AV, Bogolyubov AS. The technique for measuring the electrical conductivity of nanometer metal films in layered structures from the reflection spectra of electromagnetic radiation. Electronics. 2006;6:27-35. In: Proceedings of the Universities

[17] Usanov DA, Skripal AV, Abramov AV, Bogolyubov AS, Kalinina NV. Measurements of thickness of metal films in sandwich structures by the microwave reflection spectrum. In: Proceedings of 36th European Microwave Conference; 10-15th September 2006; Manchester, UK; pp. 509-512. DOI: 10.1109/EUMC.2006.281071

[18] Usanov DA, Nikitov SA, Skripal' AV, Ponomarev DV. Resonance features of the allowed and forbidden bands of the microwave photonic crystal with periodicity defects. Journal of Communications Technology and Electronics. 2013;58:1035-1040. DOI: 10.1134/S1064 226913110120

[19] Usanov DA, Skripal AV, Merdanov MK, Gorlitskii VO. Dielectric matrices with air cavities as a waveguide photonic crystal. Technical Physics. 2016;61:221-226. DOI: 10.1134/ S1063784216020250

[20] Maxwell-Garnett JC. Colours in metal glasses and in metallic films. Philosophical Transactions of the Royal Society. 1904;203:385-420

[21] Bruggeman DAG. Dielectric constant and conductivity of mixtures of isotropic materials. Ann. Phys. 1935;8:636-679

[22] Lichtenecker K. Dielectric constant of natural and synthetic mixtures. Physikalische Zeitschrift. 1926;4:115-158 
[23] Belyaev BA, Voloshin AS, Shabanov VF. Study of microstrip models of bandpass filters based on 1D photonic crystals. Doklady Physics. 2005;50:7-11. DOI: 10.1134/1.1862365

[24] Fernandes HCC, Medeiros JLG, Junior IMA, Brito DB. Photonic crystal at millimeter waves applications. PIERS Online. 2007;3:689-694. DOI: 10.2529/PIERS060901105337

[25] Ozbay E, Temelkuran B, Bayindir M. Microwave applications of photonic crystals. Progress In Electromagnetics Research. 2003;41:185-209. DOI: 10.1007/978-94-009-1665-4_19

[26] Saib A, Huynen I. Periodic metamaterials combining ferromagnetic nanowires and dielectric structures for planar circuits applications. Electromagnetics. 2006;26:261-277. DOI: $10.1080 / 02726340600570336$

[27] Gulyaev YV, Nikitov SA, Usanov DA, Skripal AV, Posadskii VN, Tiazhlov VS, Baykin AV. Low-dimensional waveguide microwave photonic crystals. Doklady Physics. 2014;59:437440. DOI: $10.1134 / S 1028335814100024$

[28] Usanov DA, Skripal AV, Posadskii VN, Tiazhlov VS, Baykin AV. The defect mode in a low-dimensional waveguide microwave photonic crystal. Technical Physics Letters. 2016; 42:550-552. DOI: 10.1134/S106378501605031X

[29] Cohn SB. Direct-coupled-resonator filters. Proceedings of the IRE. 1957;45:187-196

[30] Usanov DA, Skripal AV, Merdanov MK, Evteev SG. Waveguide photonic structures based on resonance diaphragms. Radioengineering. 2015;10:108-114

[31] Britun NV, Danilov VV. Photonic bandgap structures with electronically controlled characteristics. Technical Physics Letters. 2003;29:277-279. DOI: 10.1134/1.1573289

[32] Kuriazidou CA, Contopanagos HF, Alexopolos NG. Monolithic waveguide filters using printed photonic-bandgap materials. IEEE Transactions on Microwave Theory and Techniques. 2001;2:297-306. DOI: 10.1109/22.903089

[33] Usanov DA, Skripal AV, Abramov AV, Bogolyubov AS, Skvortsov VS, Merdanov MK. Waveguide photonic crystals with characteristics controlled by $\mathrm{p}-\mathrm{i}-\mathrm{n}$-diodes. Electronics. 2010;1:24-29. In: Proceedings of the Universities

[34] Kim S-I, Jang M-Y, Kee C-S, Park I, Lim H. Characteristics of microwave filters based on microstrip photonic bandgap ring structures. Current Applied Physics. 2005;5:619-624. DOI: 10.1016/j.cap.2004.08.008

[35] Kee C-S, Jang M-Y, Kim S-I, Park I, Lim H. Tuning and widening of stop bands of microstrip photonic band gap ring structures. Applied Physics Letters. 2005;86:181109. DOI: $10.1063 / 1.1906315$

[36] Usanov DA, Nikitov SA, Skripal' AV, Frolov AP, Orlov VE. Waveguides containing frame elements with electrically controlled characteristics of permitted and forbidden bands. Journal of Communications Technology and Electronics. 2014;59:1101-1106. DOI: $10.1134 / S 1064226914110230$ 
[37] Usanov DA, Skripal AV, Abramov AV, Bogolyubov AS, Skvortsov VS, Merdanov MK. Broadband waveguide matched loads based on photonic crystals with nanometer metal layers. Radioelectronics. 2009;1:73-80. In: Proceedings of the Universities

[38] Helszajn J. Passive and Active Microwave Circuits. New York, Chichester, Brisbane, Toronto: John Wiley \& Sons; 1978. 284p

[39] Lee KA, Guo Y, Stimson PA, Potter KA, Jung-Chih C, Rutledge DB. Thin-film powerdensity meter for millimeter wavelengths. IEEE Transactions on Antennas and Propagation. 1991;39:425-428. DOI: 10.1109/8.76347

[40] Usanov DA, Meshchanov VP, Skripal' AV, Popova NF, Ponomarev DV, Merdanov MK. Centimeter- and millimeter-wavelength matched loads based on microwave photonic crystals. Technical Physics. 2017;62:243-247. DOI: 10.1134/S106378421702027X

[41] Usanov DA, Skripal AV, Abramov AV, Bogolyubov AS, Skvortsov VS, Merdanov MK. Using waveguide photonic structures to measure the parameters of nanometer metal layers on insulating substrates. Electronics. 2007;6:25-32. In: Proceedings of the Universities

[42] Anlage SM, Steinhauer DE, Feenstra BJ, Vlahacos CP, Wellstood FC. Near-field microwave microscopy of materials properties. Microwave Superconductivity. 2001;375:239269. DOI: 10.1007/978-94-010-0450-3_10

[43] Usanov DA. Nearfield Scanning Microwave Microscopy and Its Applications. Saratov: Saratov University Publishing House; 2010. 100p

[44] Usanov DA, Gorbatov SS. Near Field Effects in Electrodynamic Systems with Inhomogeneities and their Application in Microwave Technology. Saratov: Saratov University Publishing House; 2011. 392p

[45] Usanov DA, Skripal' AV, Frolov AP, Nikitov SA. Microwave near-field microscope based on a photonic crystal with a cavity and a controlled coupling element as a probe. Journal of Communications Technology and Electronics. 2013;12:1130-1136. DOI: 10.1134/S1064 226913120176

[46] Usanov DA, Nikitov SA, Skripal AV, Orlov VE, Frolov AP. The patent of the Russian Federation for utility model 144869 U1 IPC The device for measuring the permittivity of plates and thicknesses of nanometer conductive films. G01N 22/00 B82B 1/00 Application: 2013125178/07 dated 05/30/2013; Pub. 10/09/2014; Bul. 25

[47] Usanov DA, Nikitov SA, Skripal' AV, Ponomarev DV, Latysheva EV. Multiparametric measurements of epitaxial semiconductor structures with the use of one-dimensional microwave photonic crystals. Journal of Communications Technology and Electronics. 2016;61:42-49. DOI: 10.1134/S1064226916010125 